\title{
RIGIDITY OF TEICHMÜLLER CURVES
}

\author{
Curtis T. McMullen
}

Let $f: V \rightarrow \mathcal{M}_{g}$ be a holomorphic map from a Riemann surface of finite hyperbolic volume to the moduli space of compact Riemann surfaces of genus $g>1$. We say $(V, f)$ is a Teichmüller curve if $f$ is a local isometry for the Kobayashi metrics on domain and range. It is well-known that $\mathcal{M}_{g}$ contains infinitely many Teichmüller curves. $^{1}$

The purpose of this note is to show:

Theorem 1. Every Teichmüller curve $f: V \rightarrow \mathcal{M}_{g}$ is rigid. Consequently $V$ and $f$ are defined over an algebraic number field.

By rigid we mean any holomorphic deformation

$$
f_{t}: V_{t} \rightarrow \mathcal{M}_{g}, \quad t \in \Delta
$$

with $\left(V_{0}, f_{0}\right) \cong(V, f)$ (and $V_{t}$ of finite volume) is trivial: we have $\left(V_{t}, f_{t}\right) \cong(V, f)$ for all $t$.

Proof. The proof combines two facts:

(1) If $X, Y$ are two hyperbolic surfaces in the Teichmüller space $\mathcal{T}_{h, n}$, and the lengths of corresponding closed geodesics satisfy $L(\gamma, X) \geq L(\gamma, Y)$ for all $\gamma$, then $X=Y$.

(2) For a fixed finite-volume hyperbolic Riemann surface $V$, and a fixed integer $g$, there are only finitely many Teichmüller curves of the form $f: V \rightarrow \mathcal{M}_{g}$.

To see (1), observe that if the lengths of corresponding closed geodesics are the same, then the same is true for geodesic currents; in particular, the Liouville current $\lambda_{X}$ (defined by the smooth invariant measure for the geodesic flow on $X$ ) has the same length on both $X$ and $Y$. But $L\left(\lambda_{X}, S\right)$ is uniquely minimized at $S=X$ [Wol], because of its strict convexity along earthquake paths; thus $X=Y$. (The same principle is used in the proof of Nielsen realization problem [Ker].)

Alternatively, one can show the Lipschitz constant of an extremal stretch map from $X$ to $Y$ is controlled by ratios of lengths of geodesics, and hence $X$ and $Y$ are isometric [Th, Thm. 8.5].

To see (2), recall that the Kobayashi metric on $V$ coincides with the hyperbolic metric (of constant curvature -4 ), and the Kobayashi metric on $\mathcal{M}_{g}$ coincides with the Teichmüller metric [Roy]. Moreover, a closed geodesic on $\mathcal{M}_{g}$ is the unique loop of minimal length in its homotopy class [Bers], and there are only finitely many closed geodesics less than a given length [Iv]. Choose a pair of closed geodesics $\alpha$ and $\beta$ on $V$

Received by the editors September 11, 2008.

2000 Mathematics Subject Classification. Primary 32G15, Secondary 37D50, 11F41.

Research supported in part by the NSF..

${ }^{1}$ In fact square-tiled Riemann surfaces $S$ are dense in $\mathcal{M}_{g}$, and any such $S$ lies on a Teichmüller curve [HS, §1.5.2]. 
crossing at a point $p$. Since $f$ is an isometry, there are only finitely many candidates for $f(\alpha)$ and $f(\beta)$, and hence for $f(p)$. Consequently there are only finitely many candidates for the isometry $f \mid \alpha$, and hence for the analytic map $f$ itself.

Alternatively, one can note that (2) follows from the geometric Shafarevich conjecture: there are only finitely many nonconstant holomorphic maps $f: V \rightarrow \mathcal{M}_{g}[\mathrm{Ar}]$, [Par]. (Here $f$ is assumed to locally lift to Teichmüller space $\mathcal{T}_{g} \rightarrow \mathcal{M}_{g}$.) For further discussion of this result, see e.g. [Mum], [Fal], or [Mc1] .

Now consider a deformation $f_{t}: V_{t} \rightarrow \mathcal{M}_{g}$ of a Teichmüller curve $(V, f)$. By the Schwarz lemma, $f_{t}$ is distance-decreasing for all $t$. Let $\gamma_{0} \subset V_{0}$ be a closed geodesic. Since $f_{0}$ is a local isometry, $f_{0}\left(\gamma_{0}\right)$ is a Teichmüller geodesic, and hence of minimal length in its homotopy class. The corresponding geodesic $\gamma_{t}$ on $V_{t}$ therefore satisfies

$$
L\left(\gamma_{t}, V_{t}\right) \geq L\left(f_{t}\left(\gamma_{t}\right), \mathcal{M}_{g}\right) \geq L\left(f_{0}\left(\gamma_{0}\right), \mathcal{M}_{g}\right)=L\left(\gamma_{0}, V_{0}\right) .
$$

Thus $V_{t} \cong V_{0}$ by fact (1) above, and then $f_{t} \cong f_{0}$ by fact $(2)$; so $(V, f)$ is rigid.

It is a standard fact that rigidity implies $(V, f)$ is defined over a number field; otherwise the transcendental elements in its field of definition would give deformations.

Finiteness. A similar argument gives the following complementary result. Let us say $f: X \rightarrow \mathcal{M}_{g}$ is generalized Teichmüller curve if $X$ is a hyperbolic Riemann surface (possibly of infinite area), and $f$ is a holomorphic, generically 1-1 local isometry for the Kobayashi metric.

Proposition 2. For a fixed genus $g$ and $L>0$, there are only finitely many generalized Teichmüller curves $f: X \rightarrow \mathcal{M}_{g}$ such that $X$ has a closed geodesic $\gamma$ of length $\leq L$.

Proof. There are only finitely many closed geodesics of length $\leq L$ in $\mathcal{M}_{g}$, so there are only finitely many possibilities for $\delta=f(\gamma)$; and $\delta$ determines $(X, f)$ up to isomorphism, by uniqueness of analytic continuation.

Corollary 3. For a fixed genus $g$ and $A>0$, there are only finitely many Teichmüller curves $f: V \rightarrow \mathcal{M}_{g}$ with area $(V) \leq A$.

Proof. An upper bound on the area of $V$ gives an upper bound for the length of its shortest closed geodesic.

A related proof, and additional finiteness results, appear in [SW].

Remark: curves in $\mathcal{A}_{\boldsymbol{g}}$. By composing with the map $\mathcal{M}_{g} \rightarrow \mathcal{A}_{g}$ sending a curve to its Jacobian, every Teichmüller curve also determines a curve

$$
J f: V \rightarrow \mathcal{A}_{g}
$$

in the moduli space of Abelian varieties.

These curves are generally not rigid, even when $J f$ is an isometry for the Kobayashi metric. Indeed, Möller has given an example in the case $g=3$ where every $X \in f(V)$ covers a fixed elliptic curve $E_{0}$, and consequently $J f(v)$ is isogenous to $B(v) \times E_{0}$ for all $v \in V[\mathrm{Mo} 2, \S 3]$. Thus the curve $J f: V \rightarrow \mathcal{A}_{g}$ can be deformed by varying the 
factor $E_{0}$. Rigidity for $J f$ under some additional hypotheses follows from [Mo1, Thm 5.1] (see also [Mo3, Cor. 6.2], which bridges a gap in the original proof).

Notes and references. For more on Teichmüller curves, their connection to polygonal billiards, and their relation to the horocycle and geodesic flows over moduli space, see e.g. [V], [KS], [MT], [Mc2], [Mo1], [Mo2], [BM].

\section{References}

[Ar] S. Arakelov. Families of algebraic curves with fixed degeneracies. Math. USSR Izv. 35(1971), 1269-1293.

[Bers] L. Bers. An extremal problem for quasiconformal maps and a theorem by Thurston. Acta Math. 141(1978), 73-98.

[BM] I. I. Bouw and M. Möller. Teichmüller curves, triangle groups and Lyapunov exponents. Preprint, 2006.

[Fal] G. Faltings. Some historical notes. In G. Cornell and J. H. Silverman, editors, Arithmetic Geometry, pages 1-8. Springer-Verlag, 1986.

[HS] P. Hubert and T. A. Schmidt. An introduction to Veech surfaces. In Handbook of Dynamical Systems. Vol. 1B, pages 501-526. Elsevier, 2006.

[Iv] N. V. Ivanov. Coefficients of expansion of pseudo-Anosov homeomorphisms. J. Soviet Math. 52(1990), 2819-2822.

[KS] R. Kenyon and J. Smillie. Billiards on rational-angled triangles. Comment. Math. Helv. 75(2000), 65-108.

[Ker] S. Kerckhoff. The Nielsen realization problem. Ann. of Math. 177(1983), 235-265.

[MT] H. Masur and S. Tabachnikov. Rational billiards and flat structures. In Handbook of Dynamical Systems, Vol. 1A, pages 1015-1089. North-Holland, 2002.

[Mc1] C. McMullen. From dynamics on surfaces to rational points on curves. Bull. Amer. Math. Soc. 37(2000), 119-140.

[Mc2] C. McMullen. Dynamics of $\mathrm{SL}_{2}(\mathbf{R})$ over moduli space in genus two. Annals of Math. 165(2007), 397-456.

[Mo1] M. Möller. Variations of Hodge structures of a Teichmüller curve. J. Amer. Math. Soc. 19(2006), 327-344.

[Mo2] M. Möller. Shimura- and Teichmüller curves. Preprint, 2005.

[Mo3] M. Möller and E. Viehweg. Kobayashi geodesics in $\mathcal{A}_{g}$. In preparation.

[Mum] D. Mumford. Curves and their Jacobians. University of Michigan Press, 1975.

[Par] A. N. Parshin. Algebraic curves over function fields. Soviet Math. Dokl. 183(1968), 524-526.

[Roy] H. L. Royden. Automorphisms and isometries of Teichmüller space. In Advances in the Theory of Riemann Surfaces, pages 369-384. Princeton University Press, 1971.

[SW] J. Smillie and B. Weiss. Finiteness results for flat surfaces: Large cusps and short geodesics. Preprint, 2008.

[Th] W. P. Thurston. Minimial stretch maps between hyperbolic surfaces. Preprint, 1998.

[V] W. Veech. Teichmüller curves in moduli space, Eisenstein series and an application to triangular billiards. Invent. math. 97(1989), 553-583.

[Wol] S. Wolpert. Thurston's Riemannian metric for Teichmüller space. J. Diff. Geom. 23(1986), 143-174.

Mathematics Department, Harvard University, 1 Oxford St, Cambridge, MA 02138-2901 